\title{
Study of Plant Waxes Using Low Temperature Method for ESEM.
}

\author{
Vilém Neděla ${ }^{1}$, Eva Tihlaříková ${ }^{1}$, Petra Schiebertová ${ }^{2}$ Iveta Zajícová ${ }^{2}$ Kateřina Schwarzerová2 \\ 1. Environmental Electron Microscopy Group, Institute of Scientific Instruments of the CAS, Brno, \\ Czech Republic \\ 2. Department of Experimental Plant Biology, Faculty of Science, Charles University, Prague, Czech \\ Republic
}

A possibility to study biological samples in their native state, or even living organisms, using an environmental scanning electron microscope (ESEM) is of great importance for biologists. Sputter-free, electrically non-conductive and wet biological samples in their living and native state are observed using ESEM under conditions of high pressure water vapors that ranges from units to thousands of $\mathrm{Pa}$ in the ESEM specimen chamber. One of unwanted consequences of the electron beam-gas interaction is the electrons scattering that results in beam spot defocusing followed by the decrease of the detected signalto-noise ratio. This can be compensated by the reduction of beam electrons gas path length and scanning speed or by the increasing of the beam energy [1]. The latter two parameters fundamentally enhance radiation damage of sensitive and soft native samples and represent a limiting factor for their observation using ESEM. This problem can be overcome by the use of special methods like Low Temperature Method for the ESEM (LTM) [2], ideally in a combination with advanced low noise detectors with very high detection efficiencies.

Wax structures, found on above-ground cell surfaces of most plants, are an example of such sensitive structures, whose shape can be studied only using a high-resolution technique. Waxes are synthesized from very long fatty acids and together with a cuticle, they form a hydrophobic barrier that prevents primarily the loss of water and exchange of gasses between the plant and the environment [3]. Cuticle and waxes are deposited on the cell wall surface of epidermal cells exposed to the environment. Waxes often form highly species-specific structures on cell surfaces [4] and contribute to specific hydrophobic properties of plant surfaces, which are important for protecting them against abiotic and biotic stresses. Apple plant leaves wax layer was observed using conventional ESEM and LTM ESEM method. Results obtained using both methods are compared and advantages of both methods are discussed.

Apple plants (Malus domestica variety Idared) were cultivated in vitro as explants on MS medium as described in [5] at $23^{\circ} \mathrm{C}$ and light period $16 \mathrm{~h}$ light / $8 \mathrm{~h}$ dark. Explants were transferred to pots with soil after root production stimulation, and cultivated further at the same light and temperature conditions. Fully developed leaves were used for the observation.

Wax structures on apple leaves were observed in the cooled and frozen state using the FEI Peltier stage equipped with a special flat cylindrical brass sample holder. Leafs were cut to $4-8 \mathrm{~mm}^{2}$ segments and placed into a drop of $2 \mu \mathrm{l}$ of water to obtain better thermal contact between the sample and the Peltier stage as well as for better sample hydration. For the case of wet state structures study (wet ESEM), the samples were observed under conditions of thermodynamic equilibrium. At the start of observation, RH was $100 \%$ and was decreased to approximately 95\% during the observation. The pressure was adjusted to $759-750 \mathrm{~Pa}, \mathrm{t}=3^{\circ} \mathrm{C}$. For the case of LTM ESEM study, the observation conditions were adjusted according to [2]: the air pressure was $650 \mathrm{~Pa}$, sample $\mathrm{t}=-20^{\circ} \mathrm{C}$. All experiments were performed on FEI ESEM QUANTA 650FEG and carried out under constant operating conditions. Wet ESEM - beam 
accelerating voltage $20 \mathrm{kV}$, probe current $36 \mathrm{pA}$, (measured in high vacuum with a Faraday cup), distance between the sample surface and the bottom surface of the GSED was $4 \mathrm{~mm}$. LTM ESEM- the beam accelerating voltage $10 \mathrm{kV}$, probe current $26 \mathrm{pA}$ and distance between the sample surface and the bottom surface of the GSED was $8 \mathrm{~mm}$.

The LTM ESEM allows the observation of samples under conditions of reduced gas pressure and low relative humidity, hence with higher resolution, see Figure 1A. This method revealed wax structures on the surface of all epidermal cells that formed linear parallel tubules (Fig. 1A, arrow). Under prolonged observation, the damage of epidermal cells sometimes resulted in the loss of turgor pressure and subsequent collapse of the cell (Fig. 1B). Such damage was very rare in the case of wet observation method, which enables to study highly hydrated native samples. Also here, wax linear tubules were observed on the surface of cells (Fig. 1C). However, hydrated samples were sometimes covered by a thin emulsion of water and/or a layer of unidentified plant material that limited the resolution of wax microstructures (Fig. 1D). We have concluded that samples were stabilized and highly resistant to the beam damage in LTM ESEM (Fig. 1A), where wax microstructures could be observed in detail. The structure of linear wax tubules was identical in wet ESEM (Fig. 1C), where the resolution was limited. To our best knowledge, this is the first description of linear wax tubules formed on the surface of expanded apple leaves using LTM ESEM. The method was demonstrated as a promising observation method suitable for studies of plant surface microstructures. Acknowledgments go to the grants [6].

\section{References:}

[1] E Tihlaříková et al, Microsc Microanal 19 (2013), p. 914.

[2] V Neděla et al, Microsc Res Techniq 78 (2015), p. 13.

[3] SB Lee et al, Plant Cell Rep 34 (2015), p. 557.

[4] SH Nguyen et al, Molecules 19 (2014), p. 13614.

[5] J Sedlák et al, Plant Protect Sci 51 (2015), p 1.

[6] The Grant Agency of the Czech Republic: grant No. GA 14-22777S, Grant of the Czech Ministry of Agriculture QJ1510353, NPUI LO1417 and LO1212 together with the European Commission (ALISI No. CZ.1.05/2.1.00/01.0017).
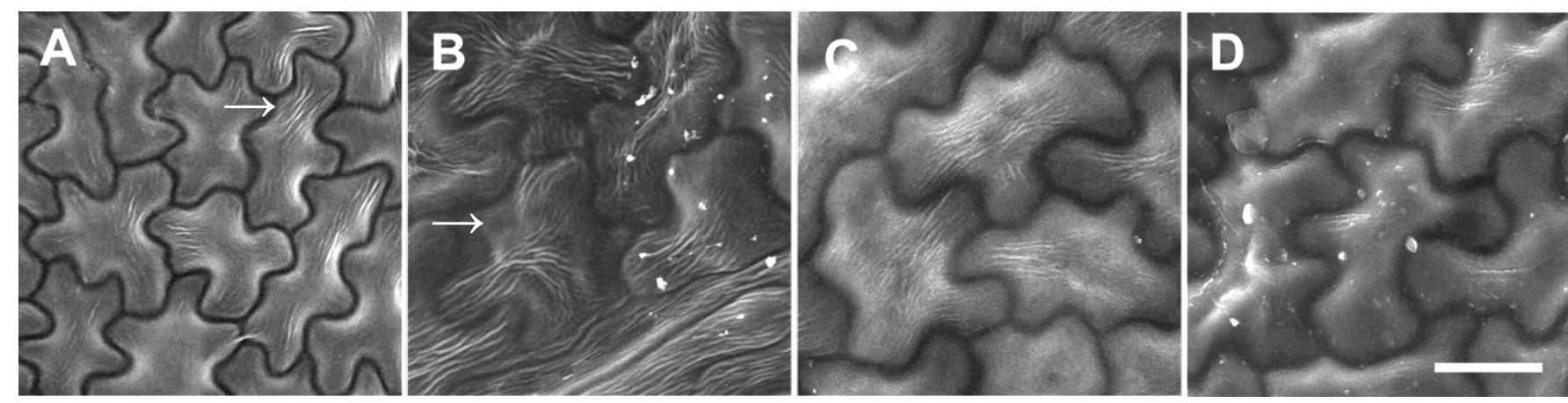

Figure 1: Wax structures on the apple leaf observed using different ESEM methods. A) The Low Temperature Method (LTM) for the ESEM shows fine wax structures (arrow). B) Prolonged observation using LTM ESEM sometimes induced cell drying and the collapse of epidermal cells (arrow). C) The wet mode of the ESEM shows fully hydrated cells, the structure of linear wax structures is identical to LTM ESEM observation. D) Wet ESEM study of waxes in a higher resolution is strongly limited, probably by the presence of a thin layer of water or plant material. Bar is $25 \mathrm{um}$. 